\title{
Actividad reflexiva en preescolares: perspectivas psicológicas y educativas
}

\author{
Reflective activity in Preschool Children: \\ Psychological and Educational Perspectives
}

Recibido: abril 5 de 2010 | Revisado: noviembre 14 de 2010 | Aceptado: enero 14 de 2011

\author{
Claudia Ximena GonzÁlez Moreno** \\ Pontificia Universidad Javeriana, Bogotá, Colombia \\ YULIA SOLOVIEVA \\ LUIS QUINTANAR ROJAS ${ }^{* * *}$ \\ Universidad Autónoma de Puebla, México
}

\section{RES U MEN}

Cómo desarrollar el pensamiento reflexivo y qué herramientas psicopedagógicas resultan más apropiadas para contribuir a tal desenlace, son asuntos que inquietan de manera permanente tanto en el campo de la Psicología como en el campo de la Educación. Numerosas investigaciones han abordado el tema de la formación del pensamiento reflexivo; sin embargo, es preocupante la brecha que existe entre las producciones científicas y su aplicación en la escuela, para mejorar los procesos de aprendizaje. Este artículo presenta un análisis reflexivo respecto a una investigación realizada desde la Psicología histórico-cultural por Vygotsky y la teoría de la actividad desarrollada por autores como Leontiev y Talizina, en donde se tenía como objetivo la aplicación del método de juego de roles en la formación del pensamiento reflexivo, en una población preescolar colombiana. El estudio es de diseño mixto, que incorpora elementos de diseño cuasiexperimental pre/post y tipo descriptivo, orientado por principios de metodología microgenética. Para ello, se trabajó con 48 niños con edades comprendidas entre los 5 y 6 años, divididos en dos grupos: control y experimental. El análisis pretest/postest permitió observar cambios importantes en la actividad escolar de los niños del grupo experimental, grupo al que se le aplicó el método de juego de roles, después de la intervención. El estudio plantea una forma de intervención innovadora para la formación gradual de la actividad intelectual por etapas dentro del aula de clase, además se constituye en una posibilidad de transformación de las prácticas pedagógicas actuales.

Palabras clave autores

Actividad reflexiva, Psicología, Educación, aprendizaje, actividad de juego, desarrollo psicológico.

Palabras clave descriptores

Psicología infantil, psicología educativa, evaluación psicológica, intervención, comportamiento infantil, características psicológicas, cognición social.

\section{A B S T R A C T}

How to develop reflective thinking and what tools are most appropriate educational psychology to contribute to that outcome are matters of concern on an ongoing basis both in the field of psychology and in the field of education. Numerous investigations have addressed the issue of the formation of reflective thought, but is concerned about the gap between scientific production and its application to the school to improve learning processes. This article presents a reflective analysis regarding the investigation carried out historical-cultural Psychology (Vygotsky) and activity theory (Leontiev, Talizina) where it was aimed at implementing the method of play role in 
the formation of reflective thinking in preschool Colombian population. The study is of mixed design, which incorporates elements of quasi-experimental pre/post and descriptive, guided by principles of microgenetic methodology. To do this we worked with 48 children aged between five and six years, divided into two groups: control and experimental. The analysis pretest/post-test allowed observing changes in the activity of school children in the experimental group, group to which he applied the method of role play, after the intervention. The study presents an innovative form of assistance to the gradual formation of intellectual activity stages within the classroom, also constitutes a possibility of transforming current teaching practices.

Key words authors

Reflection, Psychology, Education, Learning, Play Activity,

Psychological Development.

Key words plus

Child Psychology, Educational Psychology, Psychological

Evaluation, Intervention, Child Behavior, Psychological

Characteristics, Social Cognition

\section{Introducción}

La actividad reflexiva constituye una neoformación de la edad preescolar, conformando un fenómeno de la psique del niño que no existía anteriormente, es decir, que surgió como resultado de esta edad (Elkonin, 1980; Salmina \& Filimonova, 2001). En la edad preescolar, en el caso de un desarrollo positivo del niño surgen las siguientes neoformaciones psicológicas: inicio de la actividad voluntaria, la imaginación, la jerarquía de los motivos y la reflexión (Davidov, 2000; Salmina \& Filimonova, 2001). La adquisición de estas neoformaciones garantiza la preparación para el aprendizaje de nuevos conocimientos dentro de las actividades correspondientes a la etapa escolar, la escritura y la lectura, entre otras. Desde el enfoque psicológico histórico-cultural, se requiere de una metodología específica que permita desarrollar las neoformaciones de la edad preescolar.

Este artículo ofrece este tipo de metodología, a través de la evaluación que se plantea y la aplicación de un método concreto. Entre los aspectos esenciales que se abordan en este artículo, se encuentran: la caracterización de la edad psicológica de la edad preescolar, su actividad rectora y las neoformaciones psicológicas incluyendo a la actividad reflexiva, así como su eficacia preventiva referente a problemas en aprendizaje de la lectura y la escritura.
La presentación de este artículo se ha organizado en cuatro apartados. En el primero se presentan los referentes conceptuales; en el segundo se hace la descripción de la metodología; en el tercero se presentan los resultados de la aplicación del método y en el cuarto se presenta la discusión, donde se hace un análisis reflexivo desde la perspectiva psicológica y desde la perspectiva educativa; y por último se presentan las conclusiones.

\section{Referentes conceptuales}

La edad preescolar constituye una etapa importante del desarrollo psicológico del niño. En la Psicología contemporánea existen exigencias particulares para esta edad, las cuales son esenciales para la formación de los procesos de lectura y escritura. Estas exigencias se deducen de la clasificación del desarrollo psicológico del niño, realizada a partir de los trabajos de Vygotsky y se condensan en lo que se refiere a la actividad rectora y las neoformaciones (Solovieva \& Quintanar, 2005). La actividad rectora es la que determina los cambios básicos en la psique del niño en una edad dada, en la cual surgen, se forman y reconstruyen los procesos psíquicos particulares (Solovieva \& Quintanar, 2008). $Y$ las neoformaciones son nuevas formaciones que aparecen en una edad dada y que permiten nuevas formaciones en la edad siguiente (Elkonin, 1980; Salmina \& Filimonova, 2001; Solovieva, Quintanar \& Lázaro, 2006).

Al identificar las características psicológicas de los niños en la etapa preescolar, se puede determinar si corresponden con las neoformaciones propias de esa edad o si por el contrario se evidencian dificultades, las cuales deben establecer sus relaciones dinámicas y el mecanismo que subyace a ellas (Quintanar, Solovieva, Lázaro, Mejía \& Eslava, 2008). A nivel psicológico y pedagógico es ineludible resolver la causa y profundizar en la naturaleza que podría ocasionar dificultades en el aprendizaje, para comprender la forma en que están afectadas las esferas de desarrollo que constituyen la vida psíquica del niño. El análisis cualitativo de las alteraciones que se presentan durante el desarrollo, constituye un camino adecuado para determinar 
no solo qué y cómo está alterado algún proceso de aprendizaje, sino también por qué está alterado y cuál es su efecto sistémico. Sin embargo, para ello se requiere de un aparato teórico metodológico que permita el establecimiento de los diferentes niveles que se encuentran afectados (Quintanar et al., 2008).

La unidad de análisis en la teoría de la actividad planteada por Leontiev (1975) es la acción, en ella se pueden identificar todas las esferas que conforman la vida psíquica del hombre. Ninguna acción puede ser realizada por una sola función, sino que requiere de la participación de diversas funciones psicológicas, así como de las esferas cognitiva, conductual, afectivo emocional, motivacional y personalidad reflexiva. El análisis cualitativo se realiza con la ayuda de métodos especialmente elaborados para ello. Desde luego que tales métodos se fundamentan en el conocimiento acerca de la concepción de las funciones psicológicas, de su génesis y desarrollo, de su estructura mediatizada y de las regularidades de su organización (Quintanar et al., 2008).

Sin embargo, la utilización de dichos métodos no garantiza que se cumpla con el objetivo planteado: el análisis cualitativo de las dificultades. Esto solo se logra si realizamos adecuadamente el proceso de valoración y consideramos detalladamente sus resultados. Lo importante no son los resultados de la ejecución, sino el proceso de resolución de la tarea, es decir, cómo lo hace el niño. Para el análisis cualitativo de las dificultades, desde luego son necesarios los métodos especiales, pero también es importante el procedimiento o proceso de utilización de los mismos. A diferencia de las baterías estandarizadas, durante el proceso de evaluación se introducen cambios y se somete a los niños a condiciones específicas, lo que permite establecer en qué condiciones se imposibilita la ejecución y en cuáles es posible la compensación. Podemos decir que la evaluación psicológica es un proceso estructural-dinámico, donde se investiga la dinámica de las acciones alteradas (Quintanar et al., 2008).

De acuerdo a la periodización de la infancia, propuesta en la postura histórico-cultural en Psi- cología del desarrollo, cada periodo en la vida del niño tiene un contenido y objetivo particular (Quintanar \& Solovieva, 2009). Por ejemplo, el contenido mayor de la edad preescolar es la actividad de juego temático de roles, mientras que el de la edad escolar es el aprendizaje escolar dirigido. El objetivo de la etapa preescolar es la adquisición del aspecto de la actividad voluntaria del niño, que implica cuatro aspectos fundamentales: (1) tener presente el objetivo de la acción que se realiza, (2) llevar a cabo la realización de la acción sin distraerse hacía los estímulos ajenos a ella, (3) regularse por su propio lenguaje externo o por el lenguaje del adulto y (4) empezar a proponer los objetivos de las acciones propias (Quintanar et al., 2008). Tomando como ejemplo estos indicadores de la actividad voluntaria, podemos establecer indicadores de la actividad reflexiva: (1) considerar el comportamiento de los demás, logros y errores; (2) considerar el comportamiento propio, logros y errores; (3) empatía y compasión y (4) competencia (cognición) social o sentido de las relaciones sociales (LeDoux, 1996; Smith, 2002; Smith \& Mackie, 2007).

En esta investigación, el pensamiento reflexivo se define como la capacidad de reformular el propio pensamiento, mediante el uso del lenguaje, lo que lleva a reflexionar sobre el comportamiento de sí mismo y las acciones de los demás, facilitando la transformación del sentido mismo del intercambio social, habilidad compleja de la mente que impone demandas simultáneas sobre el individuo respecto a sus expresiones verbales y sus acciones voluntarias, al hacerse consciente de ellas (González Moreno, 2009). De acuerdo con los seguidores de Vygotsky (Davidov, 2000; Elkonin, 1980; Obukhova, 1995; Salmina \& Filimonova, 2001), la actividad que garantiza el pensamiento reflexivo en la edad preescolar, es el juego temático de roles sociales.

Como antecedentes investigativos, hay que mencionar que, desde hace diez años, en la Benemérita Universidad Autónoma de Puebla en México se adelantan importantes investigaciones desde el enfoque histórico-cultural. Así, se han implementado pruebas a poblaciones de prees- 
colares que asisten a jardines urbanos oficiales, urbanos privados y rurales del estado de Puebla (Lázaro, 2001; Rueda, 2001). Los resultados de estas investigaciones muestran que la falta de preparación repercute en el fracaso escolar y en los problemas de aprendizaje; así mismo, concluyen que el pensamiento reflexivo es una neoformación en la edad preescolar que facilita la preparación para la escuela (Solovieva \& Quintanar, 2008).

\section{Metodología}

\section{Diseño}

El estudio se planteó como una investigación de diseño mixto que incorpora elementos de diseño cuasiexperimental pre/post y tipo descriptivo, orientado por principios de metodología microgenética. De esta forma, se identificaron cambios cognitivos, a partir de un estado inicial, que se determinó por medio de una línea de base, para luego hacer una intervención durante cinco meses. El objetivo de la investigación fue identificar el impacto de la actividad de juego temático de roles sociales en la formación del pensamiento reflexivo en preescolares.

\section{Población}

Para el estudio, se seleccionaron 48 niños preescolares de 5 a 6 años de edad, en dos grupos, uno de control y otro experimental. Los dos grupos asistían al último nivel de preescolar (Transición) de una institución educativa preescolar regular privada.

\section{Propuesta de evaluación}

Los dos grupos participaron en un programa de evaluación inicial (pre-test) y final (post-test), mediante la Evaluación de preparación para la escuela, propuesta por Yulia Solovieva y Luis Quintanar (Quintanar \& Solovieva, 2003a, 2003b). En los dos grupos se tenía como objetivo la formación del pensamiento reflexivo; el grupo de control participó por medio de actividades lúdicas realizadas por la institución y el grupo experimental por medio del programa La utilización de la actividad de juego temático de roles en la formación del pensamiento y de la actividad reflexiva, diseñado en esta investigación (González Moreno, 2009). En la Tabla 1 se presenta la estructura de la evaluación de preparación de los niños para la escuela, de acuerdo con las neoformaciones básicas de esta edad.

TABLA 1

Estructura de la evaluación de la esfera psicológica del niño

\begin{tabular}{|c|c|}
\hline Áreas & Tareas \\
\hline Memoria mediatizada & Recordar la instrucción inicial \\
\hline Esfera voluntaria & $\begin{array}{l}\text { Lenguaje voluntario } \\
\text { Acciones voluntarias } \\
\text { Movimientos voluntarios }\end{array}$ \\
\hline Lenguaje & $\begin{array}{l}\text { Función reguladora } \\
\text { Función generalizadora } \\
\text { Función mediatizadora }\end{array}$ \\
\hline Personalidad & Actitudes y motivos \\
\hline $\begin{array}{l}\text { Habilidades matemá- } \\
\text { ticas previas }\end{array}$ & $\begin{array}{l}\text { Correspondencia } \\
\text { Igualar conjuntos } \\
\text { Seriación }\end{array}$ \\
\hline Pensamiento & $\begin{array}{l}\text { Categorización } \\
\text { Semejanzas y diferencias }\end{array}$ \\
\hline $\begin{array}{l}\text { Habilidades conversa- } \\
\text { cionales }\end{array}$ & $\begin{array}{l}\text { Turnos } \\
\text { Coherencia en el tema }\end{array}$ \\
\hline Actividad lúdica & $\begin{array}{l}\text { Juego libre } \\
\text { Juego dirigido }\end{array}$ \\
\hline
\end{tabular}

Fuente: Quintanar y Solovieva (2003b).

\section{Propuesta de intervención}

Se utilizó la actividad rectora que caracteriza esta etapa del desarrollo: el juego temático de roles sociales. El programa de intervención tuvo una duración de cinco meses, tres sesiones semanales de una hora cada una, para un total de 60 sesiones.

Durante los cinco meses del programa de intervención, las profesoras de Transición de una institución educativa lograron conocer su estructura, bases conceptuales y metodológicas. Así, las especialistas tuvieron acceso al conocimiento y uso de herramientas teóricas y procedimentales 
en la tarea de contribuir a que niños preescolares de 5 a 6 años se beneficiaran de la actividad de juego temático de roles en la formación del pensamiento reflexivo, fundamentadas desde el enfoque histórico-cultural de Vygotsky (Davidov, 2000; Elkonin, 1980). Se buscó propiciar en las profesoras reflexiones que enriquecieran sus herramientas de trabajo y les permitieran llevar a cabo una acción en el aula, razón por la que se hicieron talleres de capacitación teórico-prácticos (González, Solovieva \& Quintanar, 2009).

Se plantean tres tipos de apoyo, tanto para la evaluación como para la intervención, como se observa en la Tabla 2. El primero está relacionado con las herramientas del ambiente; el segundo, con el lenguaje verbal del adulto y el último tiene qué ver con las ayudas del niño hacia sí. Estos tipos de apoyo se derivan de un núcleo fundamental del enfoque histórico-cultural propuesto por Vygotsky (1995), denominado zona de desarrollo próximo (ZDP), el cual se refiere a la posibilidad que tiene el niño para realizar ciertas acciones y actividades, no de manera independiente, sino en colaboración con el adulto o con un par con mayor experiencia o capacidad.

TABLA 2

Tipos de apoyo utilizados

Herramientas del

ambiente

Compartido depen-

Del adulto Del niño

diente/compartido

independiente

\begin{tabular}{lll}
\hline Apoyo material & Animación & \\
Apoyo materializado & $\begin{array}{l}\text { Repetición de la } \\
\text { instrucción }\end{array}$ & Lenguaje \\
Apoyo perceptual & Muestra completa/ & verbal \\
concreto & incompleta & externo \\
Apoyo perceptual & Explicación com- & \\
generalizado & pleta/incompleta & \\
&
\end{tabular}

Fuente: elaboración propia.

Durante las sesiones de intervención, se realizaron los siguientes juegos de roles: la familia, el circo, el juego de castillos, los bomberos, la pizzería, el colegio, el museo, el almacén de computadores, el torneo de ajedrez, el partido de fútbol, el supermercado, la escuela de arte, la biblioteca, la tienda de ropa, el hospital, el restaurante, el cine, la librería, el taller de carros y la tienda de disfraces (González Moreno, 2009). En la Tabla 3 se presenta un ejemplo de cómo se hizo la intervención.

TABLA 3

Ejemplo sesión de intervención

Tarea básica Descripción

Se introduce el tema de juego de roles diciendo: "Hoy vamos a jugar a la familia". A continuación, se integra la información (1) Activa- que tienen los niños con la temática que ción de co- se va a tratar "la familia", a través de una nocimientos conversación que orienta el adulto. Se previos conversa acerca de las características físicas y formas de ser de los integrantes de la familia. Se utilizan objetos reales y se habla acerca de sus características.

(2) Planea- Se comenta con los niños qué juguetes y ción y orga- objetos se necesitan, qué es lo que hacen nización de los personajes del juego. Se toman decila actividad siones respecto a la distribución de los de juego de roles de los personajes al azar (preparar roles papelitos con los nombres de los personajes y los niños escogen los papelitos).

Se inicia el juego de roles a partir de las (3) Juego de decisiones tomadas durante la planeación roles: "La de la actividad. El adulto participa en uno familia" de los roles ayudando con propuestas concretas. (4) Análisis respecto a cómo se sintieron los niños, del juego qué les gustó y qué no. Se hacen propuestas conjuntas para la siguiente sesión.

Los niños realizan un dibujo de acuerdo al personaje que cada uno representó, el (5) Dibujo adulto también realiza un dibujo. Actividad compartida: cada uno dice qué fue lo que más le gustó del personaje.

Fuente: elaboración propia.

En la Tabla 4, se presentan algunos aspectos importantes considerados en el programa de intervención a través de la actividad de juego de roles. 


\section{TABLA 4}

Programa "La utilización de la actividad del juego temático de roles sociales en la formación del pensamiento y la actividad reflexiva en preescolares"

¿Qué pretende el programa a través del conjunto de prácticas pedagógicas que implementa?

¿Qué papel desempeña el maestro y el estudiante dentro de este proceso?
Se trata de la transformación del pensamiento de los actores que hacen parte del proceso de enseñanza-aprendizaje. Pero iqué significa esto? En cierto sentido, el pensamiento reflexivo tiene un ámbito propio de significado, se halla en el terreno de las transformaciones del pensamiento. De la sucesión coherente de incidentes y episodios e ideas a la experiencia interna consciente e intencionada y voluntaria, que capacita al individuo para dirigir sus acciones planificando objetivos y enriqueciendo la experiencia con significados.

Son agentes activos del proceso. El maestro actúa como facilitador, como guía. Por ello debe ser consciente de lo que quiere lograr en los estudiantes y cómo lo va a conseguir; de esta forma, como agente facilitador de la acción facilitación, determina el resultado de forma que éste tenga significado real con los estudiantes. Es así como, los estudiantes integran el aprendizaje con su vida cotidiana identificando sus motivaciones e intereses, los cuales se constituyen en la plataforma indispensable para orientar sus acciones. El maestro debe preguntarse: iqué hacer concretamente para satisfacer las necesidades del desarrollo psicológico en este momento de la vida del niño? y icómo lograr lo que se propone? Solo esto permitirá favorecer la esfera emocional-afectiva de los niños y garantizar su preparación para la escuela, para formar alumnos reflexivos, interesados en sus propios logros.

¿Cuál debe ser la construcción meto- Logrando que las estrategias utilizadas sean reales y naturales, interesantes, redológica más apropiada para propiciar la formación del pensamiento y la actividad reflexiva? levantes y pertinentes. Que el juego de roles sociales pertenezca al estudiante, que forme parte de un evento real, que tenga uso social, que tenga un propósito, que sea accesible y permita que el estudiante lo use.

Identificar la zona de desarrollo próximo de los estudiantes, es decir la posibilidad que tiene el niño para realizar ciertas acciones y actividades, no de manera independiente, sino en colaboración con el adulto o par, quien puede animarlo, dirigirlo, explicarle, mostrarle y enseñarle, no sólo las operaciones y su ejecución gradual, sino también otros componentes de la actividad humana, tales como el sentido, la reflexión, la compasión, la voluntad, el deseo, etc. (Vygotsky, 1978 en Solovieva, 2004). Un niño realiza una tarea conjuntamente con alguien y a continuación empieza a realizarla independientemente.

Fuente: elaboración propia.

\section{Resultados}

En la evaluación inicial o pre-test, algunos niños de los dos grupos evidenciaron fallas con la función reguladora del lenguaje, manifestándose en tareas que requerían un control constante. Estos niños presentaron dificultades para mantener el objetivo en las tareas propuestas, así como sustitución de objetivos por otros más elementales en las tareas gráficas, motoras y verbales. La ejecución pobre observada en la esfera voluntaria se evidenció también en un desarrollo insuficiente en las funciones reguladora y generalizadora del lenguaje (González Moreno et al., 2009). El desempeño en cuanto a acciones voluntarias fue bajo para los dos grupos, esto se puede atribuir a fallas de orientación visuoespacial, fallas en el reconocimiento derecha e izquierda, fallas en seguimiento de instrucciones y a fallas con la función reguladora del lenguaje. Lo anterior causa bajo desempeño escolar y debilidad en las relaciones comunicativas y, por tanto, en el concepto de sí mismo (González Moreno et al., 2009). 
En la evaluación final o post-test, en el grupo experimental la ejecución adecuada observada en la esfera voluntaria, se evidenció también en un desarrollo adecuado en las funciones reguladora, generalizadora y mediatizadora del lenguaje. En el grupo control, la ejecución pobre en la esfera voluntaria se evidenció también en un desarrollo inadecuado en las funciones reguladora, generalizadora y mediatizadora del lenguaje. Los niños del grupo control, en general, intensificaron sus fallas en la esfera voluntaria y la función reguladora del lenguaje (González Moreno et al., 2009). En el Cuadro 1, se presentan ejemplos de ejecuciones del apartado de la esfera voluntaria y de personalidad, tanto de niños del grupo control como del experimental, en la evaluación inicial y final.
Impacto de la actividad rectora de juego temático de roles sociales sobre la neoformación de la actividad reflexiva

La utilización de la actividad de juego temático de roles sociales en la formación de la actividad reflexiva, alcanzó un impacto positivo en el grupo experimental, grupo que se benefició del programa de intervención diseñado en esta investigación (González Moreno et al., 2009). Esto contribuye con la preparación de los preescolares para la adquisición de actividades complejas en la edad escolar. La intervención permitió determinar el impacto del juego temático de roles, como se observa en las Tablas 5 y 6.

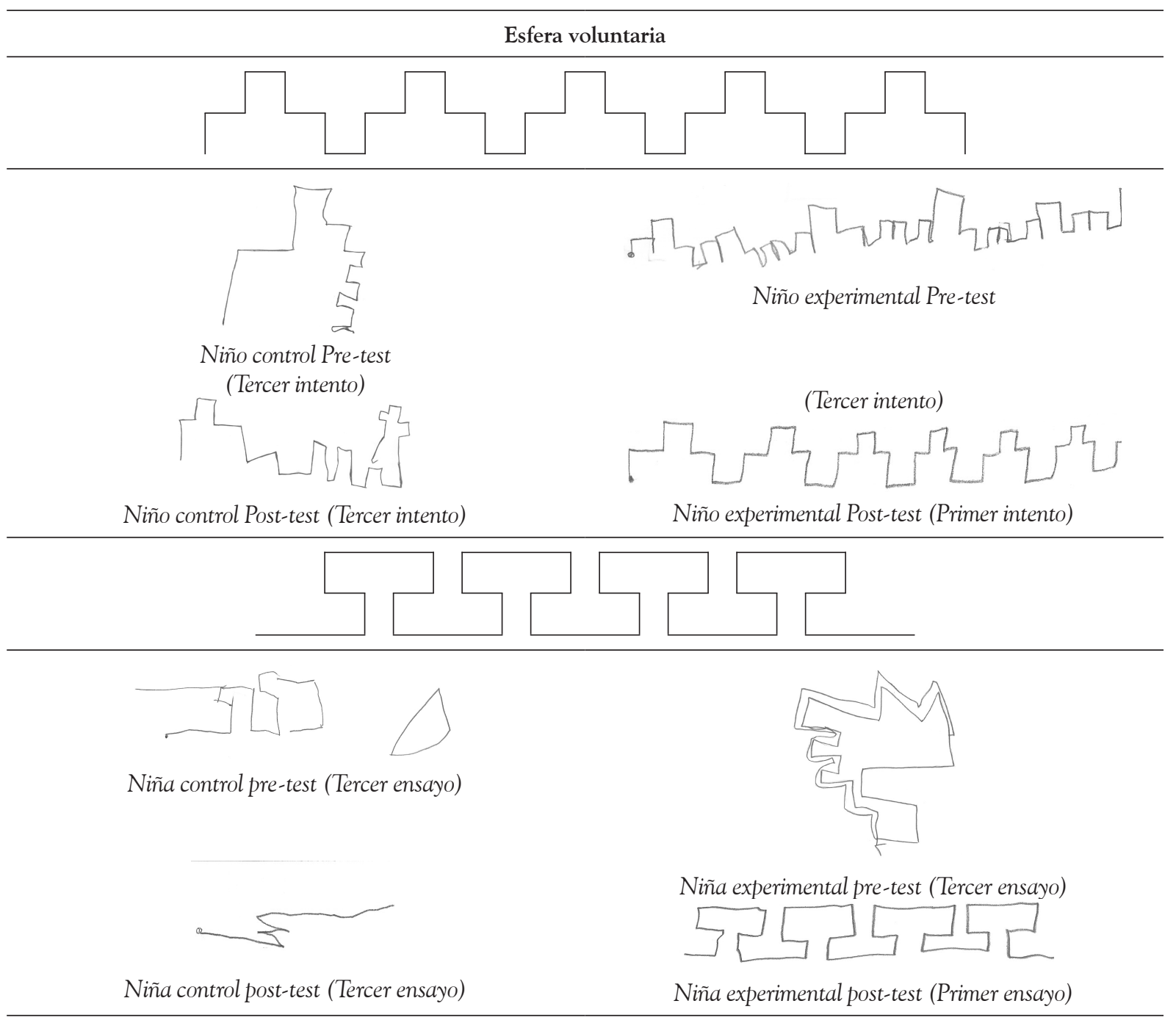




\section{Personalidad (Actitudes y motivos)}

NC. Pre-test ¿Qué es lo que más te gusta hacer?

NE. Pre-test ¿Qué es lo que más te gusta hacer?

"a mí me gusta colorear, puedo pintar, puedo hacer un avión en el "jugar con los carros, ver película y ver televisión, jugar con mi papá, Hércules. Cada vez lo veo porque mi papá es piloto" (pide una hoja con mi mamá y con mi empleada" para dibujar un avión)

NC. Post-test ¿Qué es lo que más te gusta hacer? "jugar con NE. Post-test ¿Qué es lo que más te gusta hacer? mis amiguitos, luchar, correr, mira trotar asî" Se para y empieza "jugar con mis carros". a correr por todas partes. Se le pide sentarse y pide una hoja para colorear "quiero hacer un luchador es que me vi uno que tiene poderes y rayos"

NC. Pre-test ¿Qué vas a hacer a la escuela?

NE. Pre-test ¿Qué vas a hacer a la escuela?

"en el jardín vengo a estudiar, hago más y más tareas. Me gusta di- "en el colegio de mi hermano hay parque, castillo". Se repite nuebujar. Ayer fui a visitar a mi abuelita y le llevé un regalo. Te gustan vamente y responde: "hacer las tareas".

los regalos". Se repite nuevamente y responde: "hacer las tareas, muchas tareas, muchos números y letras".

NC. Post-test ¿Qué vas a hacer a la escuela?

NE. Post-test ¿Qué vas a hacer a la escuela? "jugar, tareas de números y letras, tomar onces, comer galletas con "tareas". chocolate, cantar, luchar, luchar y luchar con mis amiguitos. No me gusta hacer tareas porque me pegan, me gusta luchar y ver a héroes voladores".

NC. Pre-test ¿Qué es lo que le gusta comer a tu papá? "come NE. Pre-test ¿Qué es lo que le gusta comer a tu papá? "sólo huepollo con arroz y huevo y salchicha y toma jugo de mora y come vos, carne, para que se engorde come mucha carne, jugo de naranja, helados de chocolate, um.. que rico y me gusta más la arepita con le toca hacer dieta. Él come tanto cada vez que chupa dedo... Mi queso y los caramelos y a mi mamá no le gusta porque me engordo prima Vivi no chupa dedo, mi primo Oscar sí". mucho y toca hacer dieta como mi tío".

NC. Post-test ¿Qué es lo que le gusta comer a tu papá?

NE. Post-test ¿Qué es lo que le gusta comer a tu papá? "sopa". "helados, dulces, chocolatinas Jet, paletas Nucita, paletas de pata de perro y ayer estuve con mi abuelita y me dio chocolate con queso y pan".

NC. Pre-test ¿Cuándo tiene miedo la gente?

NC. Pre-test ¿Cuándo tiene miedo la gente?

"Oscar tiene como 14 años, Vivi también 14 años, yo peso 58". Se "cuando sale un gato en la oscuridad y corre y corre y corre y hace repite la pregunta y responde "cuando está oscuro y me dejan miau y se come todo y se engorda". Se repite nuevamente y res- solito",

ponde: "mmm... cuando el papá me pega cuando me porto mal"

NC. Post-test ¿Cuándo tiene miedo la gente?

NE. Post-test ¿Cuándo tiene miedo la gente? "cuando salen monstruos del armario y hacen buuuu. Ayer me "cuando ve un animal carnívoro". tome un jugo y lloré porque me cai y pinté y salí y monté patineta y me dormi"

NC. Pre-test ¿Cuándo está alegre tu mamá?

NE. Pre-test ¿Cuándo está alegre tu mamá?

"yo estoy feliz cuando mi mamá me da un abrazo". Se repite la "todos los días cada vez que la asusto... Mi papá está haciendo pregunta y responde: "cuando no peleo y hago lo que ella quiere, dieta".

mañana fuimos al parque a jugar fútbol".

NC. Post-test ¿Cuándo está alegre tu mamá?

NE. Post-test ¿Cuándo está alegre tu mamá? "yo tengo que ser juicioso y no pelear y no luchar y no hacer llorar "cuando hago las tareas muy bien". al bebé". Se repite la pregunta y responde: "cuando estoy quieto y no molesto". 
NC. Pre-test ¿Cuándo estás triste?

"no me quieren".
NE. Pre-test ¿Cuándo estás triste?

"Nunca... Anoche, ayer mi papá llegó temprano y Juanpa cogió una cosa y nos empezamos a tirar agua".

NE. Post-test ¿Cuándo estás triste?

NC. Post-test ¿Cuándo estás triste?

"cuando me caí de la patineta. En el parque estuve jugando y me "cuando mi papá me pega". estrellé y lloré y comí helado y fui a visitar a mi abuelita y comí y dormi”.

Grupo control post-test

Grupo experimental post-test

- Imposibilidad para organizar la secuencia motora

- Organización de la secuencia motora

- Problema de análisis y síntesis espacial, tales como inversiones

- Impulsividad y perseveración en los trazos

- Pobre control del trazo

- Falta regulación y control

- Dificultades de freno inhibitorio

- Lentitud / impulsividad en la ejecución de movimientos

- Lentitud / impulsividad para responder preguntas

- Presentan dificultades para considerar el comportamiento propio y el comportamiento de los demás, lo confunden, no lo logran diferenciar

*NC. Niño control; NE. Niño experimental

Cuadro 1. Modelo de dictado gráfico, ejemplos de ejecución y apartado de personalidad (motivos y actitudes).

Fuente: elaboración propia.

TABLA 5

Impacto del juego temático de roles sobre la actividad reflexiva

- El paso de procesos involuntarios a procesos voluntarios. Es decir, al principio requerían del apoyo frecuente del adulto y, poco a poco, este se fue reduciendo.

- La regulación consciente de la actividad. En un primer momento con apoyo del adulto, quien la orientaba y organizaba utilizando herramientas del ambiente, de forma compartida dependiente a la forma compartida independiente.

- La formación de motivos e intereses por parte de los niños. Al iniciar las sesiones de intervención, los niños esperaban a que el adulto asignara los roles, en la sesión 24 los niños, en general, empezaron a solicitar algunos roles. Desde ese momento, ellos elegían cuál rol querían asumir.

- Garantizar el comportamiento mediatizado, es decir, la posibilidad de utilizar diferentes signos y símbolos como medios de organización externa e interna de la actividad.

- Impulsar el desarrollo de la imaginación, porque los niños en cada una de las sesiones debían imaginar los personajes, sus roles, comportamientos, reglas y objetos.

- La formación del objetivo de la actividad individual y compartida.

- Conocer la esencia de las relaciones sociales: el sentido mismo del intercambio social. Esto se evidenció cuando los niños tenían que ser amables y estar atentos a la solicitud de sus compañeros.

- Reflexionar sobre el comportamiento propio y las acciones de los demás.

- Se generó una necesidad social y cognoscitiva de participación genuina en los diferentes juegos de roles por parte de los niños. Esto permitió gradualmente inducir en la actividad el objetivo o propósito, para lo cual fue necesario lograr que los niños comprendieran en qué consistían las acciones y las planearan, utilizando herramientas del ambiente.

- Modificar el concepto que los niños tenían acerca de sí mismos, porque se dieron cuenta de que podían realizar varias acciones, al principio de forma compartida dependiente del adulto y después de forma independiente.

- Alcanzar un efecto emocional positivo en los niños. 
- Crear una zona de desarrollo próximo que, a lo largo de las sesiones de intervención, se convirtió en zona de desarrollo real en los niños.

- El uso de herramientas del ambiente condujo al desarrollo de una actividad intelectual mediatizada que produjo cambios en el comportamiento de los niños: regulación y autocontrol.

- Mejoramiento en la actitud hacia el aprendizaje.

- Aumento en la participación en clase.

- Interés y motivación amplia frente a la actividad escolar.

- Generar vínculos sociales entre los niños.

- Desarrollar la habilidad para trabajar conjuntamente en busca de un objetivo.

- Incrementar la riqueza léxica.

- Favorecer el desarrollo de dos etapas fundamentales del pensamiento reflexivo: (1) de acciones materializadas y perceptuales generalizadas y (2) de lenguaje verbal externo. Ahora los niños regulan sus acciones a través del uso del lenguaje verbal externo, es decir que hablan mientras realizan la actividad, lo cual les permite planear y organizar lo que hacen. - Desde el inicio de la investigación, en la evaluación inicial se le dio la posibilidad a los niños de que se dieran cuenta que podían realizar las tareas propuestas con ayuda. Este simple hecho marcó un cambio radical en la percepción de ellos mismos. Cuando se daban cuenta de que ahora podían hacer lo que antes era prácticamente imposible, empiezan a interesarse en las tareas escolares, aumentando su motivación hacia dichas tareas. La realización de las tareas cambia la percepción de frustración por satisfacción, lo cual tiene incidencia importante en el desarrollo de su personalidad. Ya en la evaluación final, se evidenciaron cambios significativos en el grupo experimental porque ahora los niños son más independientes en la realización de acciones. Además logran ejecutar de forma independiente lo que en la evaluación realizaban de forma compartida y con apoyo del adulto.

Fuente: elaboración propia.

TABLA 6

Acerca del impacto de los resultados

\begin{tabular}{|c|c|}
\hline Apartados & Descripción \\
\hline $\begin{array}{l}\text { En la prepa- } \\
\text { ración es- } \\
\text { colar }\end{array}$ & $\begin{array}{l}\text { - Los niños del grupo experimental lograron establecer las neoformaciones básicas de esta edad: conducta } \\
\text { voluntaria y actividad reflexiva. Esto indica que pasaron exitosamente por esta edad y están listos para } \\
\text { pasar a la edad siguiente, la edad escolar. }\end{array}$ \\
\hline
\end{tabular}

- La actividad escolar exige un nivel de desarrollo suficiente del comportamiento voluntario, de la motivación y de ciertos conocimientos previos que a través de la utilización de la actividad de juego temático de roles sociales, se pudo desarrollar

- La actividad escolar llevó al planteamiento de la acción mediatizada por instrumentos materiales o En la activi- instrumentos psicológicos para alcanzar la asimilación y apropiación de nuevos conocimientos y habidad escolar lidades. Aquí, activar conocimientos previos utilizando apoyos materiales y perceptuales generalizados, condujo a la comprensión de nuevos conocimientos y por consiguiente a lograr su apropiación y dominio utilizando apoyos materializados y perceptuales generalizados.

- Esta investigación permitió evaluar a los niños que ingresan a la primaria, determinar su nivel de preparación y brindar apoyo interventivo dentro del aula de clase.

- Este estudio plantea la reflexión en torno a que es más importante centrarse en el desarrollo de las

En las neoformaciones en la edad preescolar neoformaciones de la edad preescolar y evaluar el grado de su formación, que iniciar la enseñanza de la lectura y la escritura sin tener las bases para su adquisición porque esto conduce al fracaso escolar y produce un efecto emocional negativo.

- El lenguaje pasó de cumplir una importante función en las relaciones sociales, al transformarse en un instrumento de la organización psíquica interna del niño. 


\begin{tabular}{ll}
\hline \multicolumn{1}{c|}{ Apartados } & Descripción \\
\hline & $\begin{array}{l}\text { Mediante la acción intelectual de pensar reflexivamente se evidenciaron mecanismos del sujeto para } \\
\text { transformar su comportamiento y el de los demás: procesos de planeación, verificación y control. Estas }\end{array}$ \\
$\begin{array}{ll}\text { En la activi- } \\
\text { dad reflexiva }\end{array}$ & $\begin{array}{l}\text { acciones intelectuales adquirieron una fuerza impulsadora propia de realización de nuevas acciones. } \\
\text { la motivación e interés. }\end{array}$ \\
\hline En las accio- & - Los niños alcanzaron la organización externa de la acción, apoyándose en su propio lenguaje verbal \\
nes mentales & externo, así como la planificación verbal desplegada y completa.
\end{tabular}

- A través de esta actividad, se dio la posibilidad para que los niños asumieran ciertos papeles por la propia voluntad, se sometieran a algunas reglas y conocieran la esencia del sentido mismo del intercambio social.

- El juego temático de roles sociales le dio la oportunidad a los niños de darse cuenta de algunas relaciones que existen en la sociedad y de percibir conscientemente las reglas, las obligaciones y deberes

En el juego temático de roles sociales que se deben cumplir. El hecho de participar en situaciones creadas contextualmente les ofreció a los niños la posibilidad de representar y comprender aquellas situaciones reales de las que forma parte, en la vida cotidiana. Aspectos indispensables en esta edad, los cuales permiten el desarrollo de las neoformaciones siguientes, debido a que son sujetos de adquisición de contenidos específicos de acciones intelectuales y de actividades complejas en la edad escolar (Solovieva \& Quintanar, 2005).

- La comprensión de las diferentes tareas en los juegos de roles llevó a dominar nuevos procedimientos de acción, y por consiguiente a realizar la transferencia a situaciones nuevas.

- El juego de roles permitió que los niños en edad preescolar respetaran los roles, tomaran turnos al actuar y hablar, planearan y regularan la actividad, siguieran instrucciones.

- El enfoque histórico-cultural se constituye en una aproximación para la prevención de dificultades en el aprendizaje y la promoción de habilidades escolares y de toda la psique humana.

- La evaluación y el diagnóstico desde el enfoque histórico-cultural permite identificar la causa de las dificultades en el aprendizaje escolar y, por tanto, tomar las medidas correctivas necesarias.

En el enfoque histórico-cultural

- Se resalta la importancia de la zona de desarrollo próximo durante la aplicación de la evaluación, debido a que gracias a ella se pudo determinar el nivel de desarrollo actual y potencial en cada niño y en los grupos.

- Los niños de la población estudiada no contaban con las bases necesarias para la preparación escolar. El objetivo de la investigación fue garantizar esas bases. La inclusión de actividades a través del programa de intervención resultó ser efectiva.

- En la investigación, se evidenció que los resultados en la intervención dependen de las elecciones que los agentes o actores hagan para alcanzar objetivos, de su intencionalidad, de las herramientas o estrategias que se utilicen. Para ello, fue indispensable que las maestras fueran conscientes respecto a qué querían lograr en los niños y cómo lo iban a conseguir, de esta forma, ellas, como agentes facilitadores de la acción facilitación, determinaron el resultado de forma que este tuviera significado real con los niños para que lo lograran transferir a diferentes situaciones y contextos.

En el rol del

- El adulto cumplió un rol muy importante en la formación de la actividad reflexiva, al explicar cómo y en qué orden era necesario ejecutar una acción, permitió verificar la posibilidad de ejecución de la acción utilizando apoyos materiales, materializados, perceptuales concretos y perceptuales generalizados. Esto posibilitó que los niños se preguntaran por el significado de sus acciones respectivas así como por el significado de las acciones de los demás y les ayudó en la planificación de acciones.

formacion de

la actividad reflexiva

- En las acciones pedagógicas que realiza el maestro, fue importante preguntarse iqué hacer? y icómo lograr lo que se propone? pensando en que los niños pudieran hacerlo, de esta forma los niños estuvieron interesados porque se dieron cuenta que son capaces.

- Las maestras utilizaron estrategias de enseñanza que implicaron la orientación desplegada.

- Las maestras consideraron la participación emocional de los niños y la empatía entre ellos. Para esto utilizaron preguntas como: ¿Quién lo hizo mejor? ¿Cómo podemos ayudar? ¿Qué te gusta? ¿Qué no les gusta?, etc. 


\begin{tabular}{|c|c|}
\hline Apartados & Descripción \\
\hline & 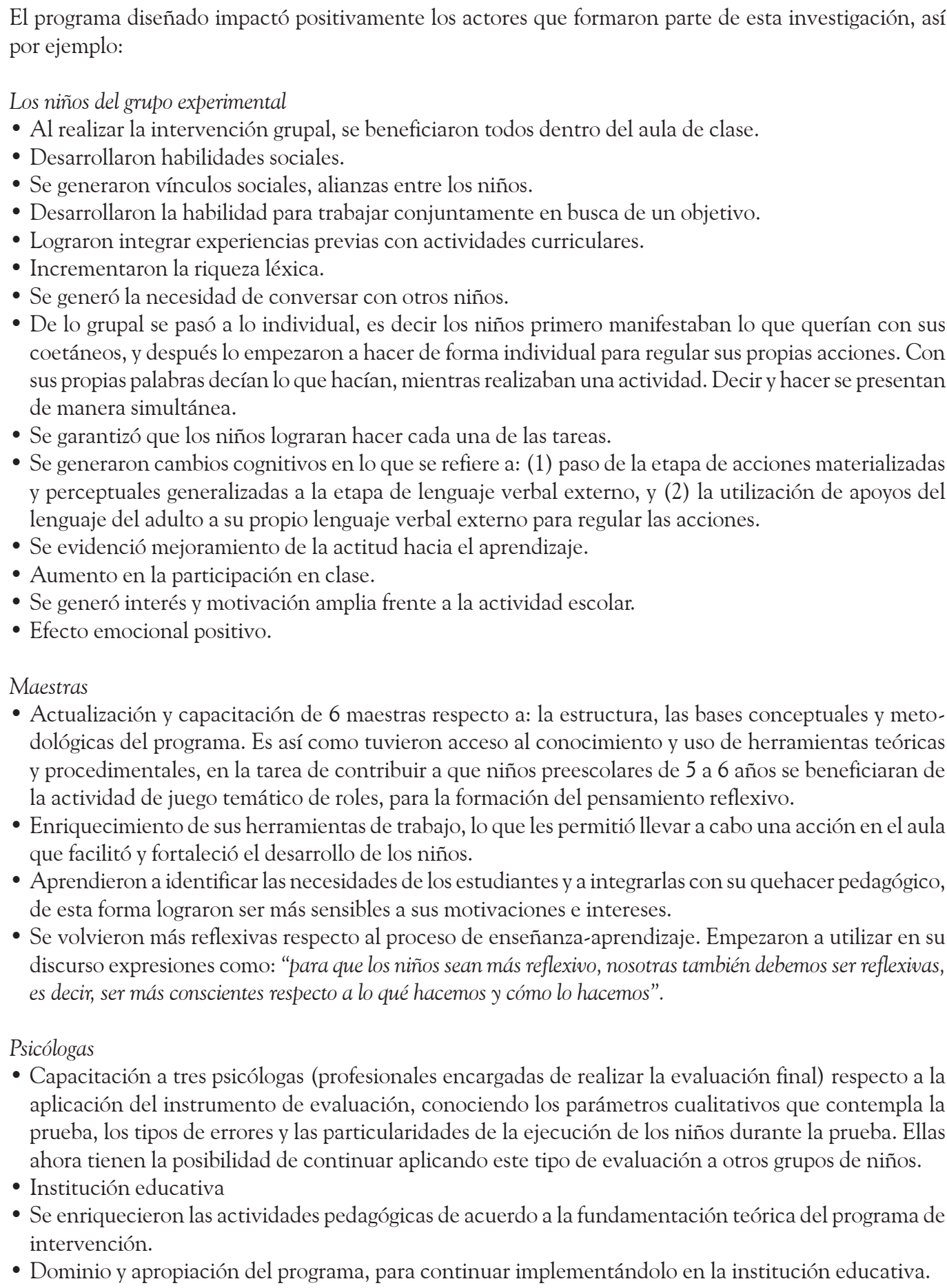 \\
\hline
\end{tabular}




\begin{tabular}{|c|c|}
\hline Apartados & Descripción \\
\hline $\begin{array}{l}\text { For } \\
\text { los } \\
\text { ma }\end{array}$ & $\begin{array}{l}\text { Programa de evaluación } \\
\text { - Identifica las características psicológicas de la edad preescolar. } \\
\text { - Identifica si las neoformaciones corresponden con la edad preescolar y si los niños están listos para } \\
\text { pasar a la edad siguiente. } \\
\text { - Identifica la zona de desarrollo real y la zona de desarrollo próximo de los niños (Vygotsky, 1995). } \\
\text { Programa de intervención } \\
\text { - Es agradable para los niños y es efectivo para que ellos logren identificar sus motivaciones e intereses. } \\
\text { Eleva la motivación intrínseca. } \\
\text { - Es un programa organizado y estructurado que fácilmente se puede adaptar a un grupo poblacional. } \\
\text { - Alto grado de sistematización en los procesos de evaluación e intervención. }\end{array}$ \\
\hline & $\begin{array}{l}\text { - Esta investigación presenta aportes tanto para la psicología como para la educación, al mostrar clara- } \\
\text { mente: (a) la transformación de las acciones intelectuales en los niños, que pasan de la utilización de } \\
\text { objetos materiales a su representación, de imágenes a su representación, primero en el plano compartido } \\
\text { dependiente y después al plano compartido independiente, del apoyo verbal externo propio a la reali- } \\
\text { zación independiente de acciones y (b) el proceso de transferencia del programa: acciones materiales } \\
\text { a acciones intelectuales, en donde el programa "La utilización de la actividad de juego temático de } \\
\text { roles sociales en la formación del pensamiento reflexivo en preescolares" podría decirse, se constituye } \\
\text { en motor y plataforma para la preparación escolar. }\end{array}$ \\
\hline $\begin{array}{l}\text { Implicacio- } \\
\text { nes prácticas }\end{array}$ & $\begin{array}{l}\text { - Esta investigación da la posibilidad de implementar el programa en instituciones educativas públicas } \\
\text { y privadas. } \\
\text { - La evaluación se puede realizar a niños desde Transición hasta Segundo de Primaria. La intervención } \\
\text { podría ser usada con niños de una amplia gama de edades. } \\
\text { - De esta investigación, podrían surgir otras investigaciones en donde se desarrollen las siguientes etapas } \\
\text { del pensamiento reflexivo (González Moreno, 2009). }\end{array}$ \\
\hline
\end{tabular}

Fuente: elaboración propia.

\section{Discusión}

\section{Análisis reflexivo desde la perspectiva psicológica}

Los resultados muestran que la actividad de juego de roles puede tener efectos importantes no sólo en la esfera cognoscitiva, sino sobre todas las esferas de la psique infantil: la esfera emocional-afectiva, de motivos e intereses, comportamental y de personalidad reflexiva. Todas ellas constituyen una unidad inseparable e indispensable para el éxito escolar del niño, el cual constituye el objetivo psicológico del desarrollo en la edad preescolar (Lázaro, Solovieva, Cisneros \& Quintanar, 2009; Solovieva \& Quintanar, 2008; Talizina, 2000). Estos resultados concuerdan con otros obtenidos al aplicar la acti- vidad de juego en grupos de niños preescolares en México (García, 2005; Lázaro et al., 2009).

El juego de roles, como actividad rectora en la etapa preescolar, en la cual los niños participan asumiendo diferentes papeles, permite el desarrollo de las neoformaciones básicas de esta edad: conducta voluntaria y pensamiento reflexivo con sus dos primeras etapas: (a) acciones materializadas y perceptuales generalizadas y (b) lenguaje verbal externo. Estos elementos en conjunto se constituyen en requisitos para la preparación de los niños para la escuela, así como para la adquisición de actividades complejas en la edad escolar. En la Tabla 7 se presenta la descripción de las etapas del pensamiento reflexivo en la edad preescolar y sus características. 


\section{TABLA 7}

Etapas, condiciones requeridas y características de la actividad reflexiva en preescolares

\begin{tabular}{|c|c|c|}
\hline Etapa & $\begin{array}{l}\text { Condiciones } \\
\text { requeridas }\end{array}$ & Característica \\
\hline $\begin{array}{l}\text { Etapa de } \\
\text { acciones ma- } \\
\text { terializadas y } \\
\text { perceptuales } \\
\text { generalizadas }\end{array}$ & $\begin{array}{l}\text { * Se requiere de la } \\
\text { internalización del } \\
\text { las características } \\
\text { de los objetos ma- } \\
\text { teriales. } \\
\text { * Se realiza con crea- } \\
\text { tividad simbólica. } \\
\text { * Actividades com- } \\
\text { partidas. }\end{array}$ & $\begin{array}{l}\text { * Con apoyo del adulto, los niños empiezan a identificar las características so- } \\
\text { bresalientes de los objetos del ambiente: forma, color, tamaño, textura, etc. } \\
\text { Poco a poco, pasan del plano material (objetos reales) al materializado (repre- } \\
\text { sentación de esos objetos), y del perceptual concreto (imágenes) al perceptual } \\
\text { generalizado (representación de esas imágenes). } \\
\text { * Representan de forma interna las características esenciales y diferenciales de } \\
\text { los objetos, considerando que estas se desarrollan y forman en la actividad } \\
\text { objetal. } \\
\text { * Logran identificar un objetivo en la acción con apoyo del adulto, quien utiliza } \\
\text { herramientas del ambiente: apoyos materiales, materializados, perceptuales } \\
\text { concretos y perceptuales generalizados. De esta forma orientan su actividad. } \\
\text { * Utilizan objetos reales y hablan acerca de sus características. } \\
\text { * Utilizan la representación de los objetos mientras realizan actividades gráficas } \\
\text { como dibujar. } \\
\text { * Utilizan imágenes para expresar las características de lo que observan. } \\
\text { * Utilizan representaciones de esas imágenes. }\end{array}$ \\
\hline $\begin{array}{l}\text { Lenguaje ver- } \\
\text { bal externo }\end{array}$ & $\begin{array}{l}\text { Se requiere de: } \\
* \text { Actividades com- } \\
\text { partidas. } \\
* \text { Experiencias. } \\
* \text { Conversación. }\end{array}$ & $\begin{array}{l}\text { * Repiten lo que escuchan. } \\
\text { * Hablan mientras realizan una actividad. } \\
\text { * Dicen lo que saben. } \\
\text { * Hablan de lo que conocen. } \\
\text { * Preguntan para confirmar qué deben hacer. } \\
\text { * Repiten la instrucción que se les da y/o la preguntan qué se hace (completa o } \\
\text { una parte) para confirmar lo que deben hacer. } \\
\text { * La formación de la acción está basada en el lenguaje verbal externo con apoyo } \\
\text { de objetos materiales. Poco a poco, se va reduciendo la utilización de repre- } \\
\text { sentaciones gráficas para pasar al lenguaje verbal externo. De esta forma el } \\
\text { lenguaje puede verse como una forma material de la acción. } \\
\text { * Logran planear sus acciones utilizando el lenguaje verbal externo. } \\
\text { * Utilizan expresiones como "voy a hacer...". } \\
\text { * Se autocorrigen. }\end{array}$ \\
\hline
\end{tabular}

Fuente: elaboración propia.

\section{Análisis reflexivo desde la perspectiva educativa}

El presente estudio plantea la reflexión en torno a la actividad escolar, la cual exige un nivel de desarrollo suficiente del comportamiento voluntario, de la motivación y de ciertos conocimientos previos que, con la utilización de la actividad de juego temático de roles, se puede alcanzar (González Moreno et al., 2009). Los resultados afirman las ideas de Mijail Bajtin (1997) acerca de que el pensamiento reflexivo solo se forma por la inte- racción con los demás: el niño aprende a verse a sí mismo a través de los ojos de los demás. Los resultados señalan la importancia de la consideración de los métodos que permitan desarrollar no solo la actividad cognitiva, sino también la actividad reflexiva, para garantizar el grado necesario de las neoformaciones de la edad preescolar, antes de iniciar la enseñanza formal de la lectura y la escritura. Frecuentemente, la formación de la lectoescritura en la escuela primaria se realiza sin tener las bases para su adquisición, y esto conduce al fracaso escolar y produce un efecto emocional negativo (Solo- 
vieva \& Quintanar, 2008). Infortunadamente, en las instituciones educativas preescolares actuales se pierde tiempo valioso, centrando el interés solo en la formación de los hábitos aislados de lectura y escritura, considerando al juego solo como una forma de actividad recreativa y adicional. Se pondera la cantidad de conocimientos más que la calidad. No se presta ninguna importancia a la formación de la actividad voluntaria y reflexiva de los niños preescolares. Por ello, con frecuencia, la escolaridad presenta fallas en la comprensión lectora, en la producción de textos y dificultades en procesos matemáticos. Cabe señalar que no es posible garantizar el pensamiento conceptual lógico sin esta característica esencial de la actividad que es su aspecto reflexivo. Sin reflexión no hay posibilidad de darse cuenta de sus propios logros y fracasos, del sentido de las acciones y los problemas tanto en el ámbito educativo, como de la vida en general.

Si se desarrollan adecuadamente las neoformaciones de esta etapa del desarrollo, se abren posibilidades cognoscitivas amplias para el niño. Así mismo, se contribuye a desarrollar los conocimientos acerca de su propio idioma, formar una actitud de reflexión no solo hacia el lenguaje y el idioma, sino también hacia su propio ser. Y de esta forma el niño adquiere la posibilidad de comunicarse con la cultura humana general que traspasa fronteras.

Cuando se habla que un estudiante ha aprendido algo, es porque ha habido un cambio en sus destrezas, habilidades y actitudes que se reflejan en un cambio de comportamiento. Es así como la formación de un pensamiento intencionado, reflexivo y orientado hacia objetivos, es una exigencia de la sociedad actual, porque hablar de pensamiento reflexivo nos remite al concepto de pensar para aprender, teniendo en cuenta un individuo intencional, autónomo e independiente y, en consecuencia, responsable de su propio aprendizaje. En este proceso dinámico, los maestros generan conflicto cognitivo en el niño, lo que permite transformar sus esquemas preexistentes en otros nuevos que incorporan los nuevos aprendizajes, estableciendo relaciones entre ellos.

Solo sobre la base del desarrollo previo de la actividad reflexiva, el pensamiento reflexivo en la escuela se convierte en un reto que le permite al estudiante aprender a usar sus habilidades intelectuales, a definir sus propios procesos de pensamiento, a determinar en qué tipos de situaciones son aplicables y, en esta medida puede llevarlos a diferentes contextos.

El papel del pensamiento reflexivo en la educación, significa, en primer lugar, comprender que las actuaciones de los actores involucrados deben ser conscientes. En segundo lugar, si se acepta que la escuela contribuye a estructurar formas de vida social, no puede desarticular de sus prácticas educativas la producción y organización de la experiencia de sus actores, en especial de los estudiantes. Eso significa que la escuela debe estar comprometida con la comprensión de la experiencia, hecho que implica reconocer e integrarse a la multiplicidad de voces que circular en la vida social y, en consecuencia, construir los principios desde los cuales es posible comprenderlos y analizarlos (Pardo, 2000).

Pensar y actuar reflexivamente implica pensar sobre qué y cómo se piensa; estas acciones tienen incidencia importante en el comportamiento del individuo y por consiguiente en su desempeño. Un individuo que piensa y actúa reflexivamente sabe en qué momento y cómo utilizar sus habilidades y destrezas tanto en el contexto educativo como en el social, familiar, nacional, etc.

En el ámbito educativo, pensar reflexivamente se convierte en un reto. Se trata de la sucesión coherente de incidentes e ideas a la experiencia interna consciente e intencionada y voluntaria, que capacita al individuo para dirigir sus acciones planificando objetivos y enriqueciendo la experiencia con significados. Aquí surge la pregunta ¿cómo lograr que los estudiantes empiecen a pensar reflexivamente? De acuerdo con esta investigación, esto se puede lograr haciendo explícitas las estrategias que utilizan para pensar, comunicarse, conversar, interactuar y aprender en la interacción social, a través de las actividades compartidas en la experiencia, utilizando apoyos materiales, materializados, perceptuales concretos y perceptuales generalizados.

La formación de la actividad reflexiva influye positivamente sobre varios aspectos de la vida de 
los individuos y sociedades, dado que mediatiza el crecimiento en valores y el aprendizaje de actitudes civilizadas como la solidaridad, la tolerancia, la participación, la equidad y el respeto por la vida, facilita la negociación en situaciones de conflicto, mejora el desempeño en lo académico y en el trabajo. Así mismo, el pensamiento y la actividad reflexiva, ayuda a construir mejores seres humanos: personas civilizadas, éticas, solidarias, más humanas y comprometidas en la construcción de una sociedad más inclusiva. En este sentido, los individuos que se expresan bien, piensan mejor y están en capacidad de participar legítimamente en una democracia porque pueden expresar opiniones de manera convincente y clara; así mismo, logran tomar decisiones con mayor facilidad y, por consiguiente, resuelven problemas de forma eficiente.

La invitación es entonces a reflexionar, como afirma Antonio Arellano (2005), acerca del quehacer educativo en el complejo y fortuito mundo de hoy, en el cual la educación y los sistemas escolares han mostrado su dificultad para acoplarse al espíritu de la época y su fragilidad para enfrentar los retos de la sociedad global, y más bien han instrumentalizado sus dinámicas, marginando el encuentro humano en este proceso de transmisión y recreación de los saberes culturales. Siguiendo esta línea de pensamiento, Esteban Ocampo (1997) afirma que es indispensable pensar sobre la acción misma de quienes realizan actividades que se dirigen a la formación de otros, en donde es necesario alcanzar el dominio de lo ético, el reconocimiento de los otros como posibilitadores de vida, de existencia, de saber, de hacer y a nosotros mismos como sujetos con capacidad de revertir a los demás aquello que es nuestra propia vida. En este sentido, nuestra investigación muestra que si el maestro piensa la acción misma de juego temático de roles sociales, éste se constituye en un método real aplicable que permite adquirir la actividad voluntaria y reflexiva en la edad preescolar de manera natural y paulatina. En semiótica, se define el juego de la situación de conflicto, análogo de la situación real de conflicto, que posee una situación interna jerárquica de valores (Lotman, 2003), es decir, se trata de un conflicto en el que se "juega" o se "ensaya" sin convertirse en un conflicto real. A esta definición semiótica, podemos agregar un componente psicológico diciendo que el juego es una situación que modela conflicto de acuerdo a ciertas reglas de comportamiento: estas reglas son las reglas de juego. De acuerdo a Elkonin (1980), en la actividad de juego temático de roles, la regla es el respeto del rol aceptado por el niño. El hecho de aceptar un rol prepara perfectamente para los inicios de la actividad reflexiva.

\section{Conclusiones}

No es necesario esperar hasta que los niños presenten dificultades escolares, sino que es posible identificar tempranamente los aspectos débiles en el desarrollo que pueden generar esas dificultades. Esto se puede hacer a través de la evaluación que se propone en esta investigación, lo cual conduce a la elaboración de programas preventivos, utilizando el juego de roles como actividad rectora característica de la edad preescolar.

Para la implementación de los programas, es indispensable la preparación teórica y metodológica desde la Psicología histórico-cultural, así como de conocimientos de la Psicología del desarrollo y de la Psicología educativa.

El enfoque histórico-cultural ofrece una metodología clara y aplicable a las condiciones de países de América Latina: actividad rectora de juego de roles sociales.

El análisis psicológico a partir de la aproximación histórico-cultural y la teoría de la actividad, implica la valoración cualitativa de las formaciones psicológicas de la edad preescolar, es decir, de la actividad de aprendizaje. Por ello, es indispensable que el maestro establezca cuáles son las acciones escolares accesibles y cuáles son particularmente difíciles para el niño, y busque las vías psicológicas y psicopedagógicas para su solución (Quintana, Solovieva, Lázaro \& Bonilla, 2008). En el Cuadro 2 , se presenta la relación entre las perspectivas psicológicas y educativas. 


\section{CUADRO 2}

La actividad reflexiva a través de las perspectivas psicológicas y educativas

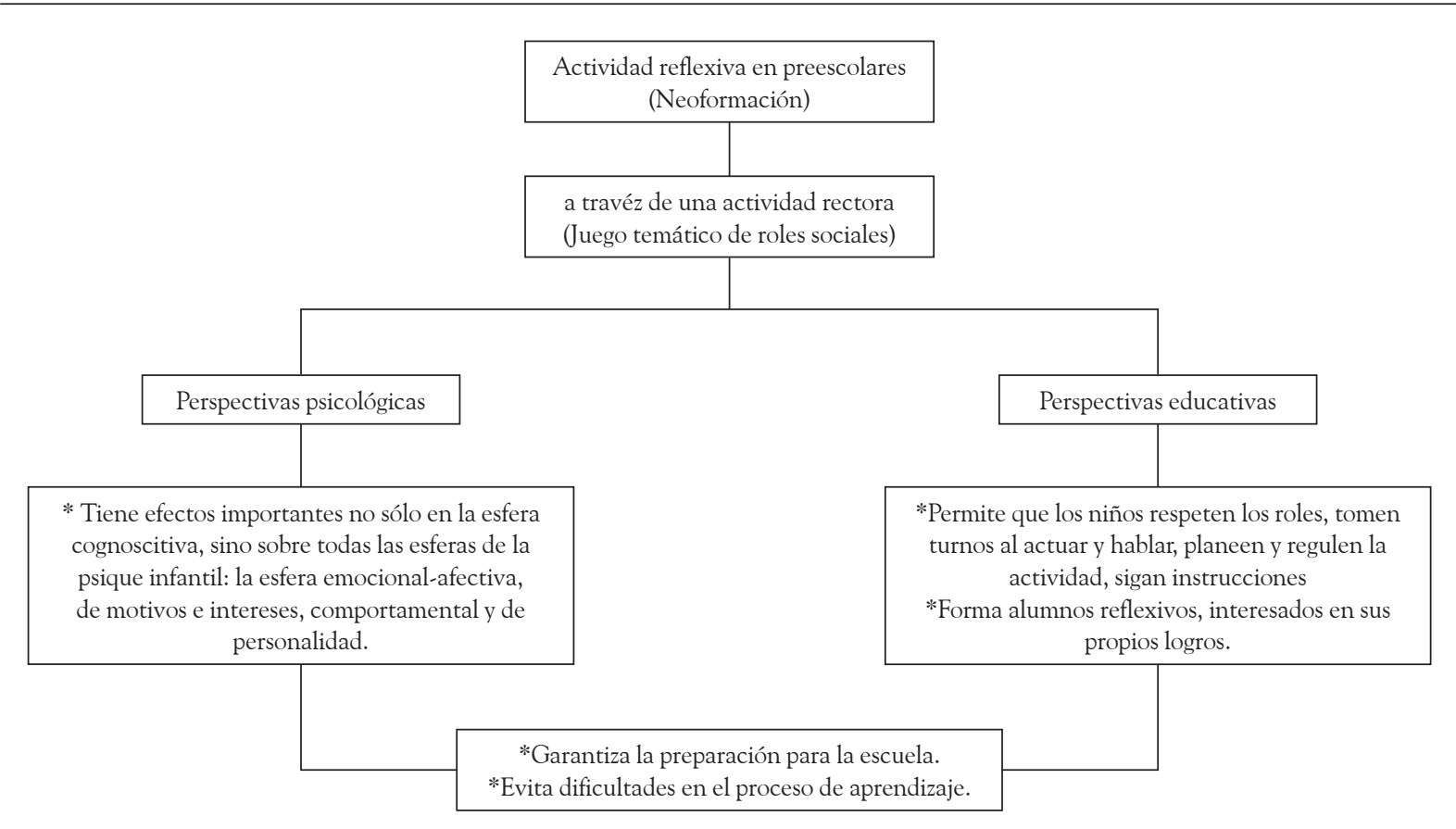

Fuente: elaboración propia.

\section{Referencias}

Bajtin, M. M. (1997). Obras escogidas. Obras de 1940 - inicios de 1960 (Vol. 5). Moscú: Diccionarios Rusos.

Davidov, V. V. (2000). Tipos de generalización de la enseñanza. Moscú: Sociedad Pedagógica de Rusia.

Elkonin, D. B. (1980). Psicología del juego. Madrid: Pablo del Río.

García, M. A. (2005). El uso de las actividades de juego y cuento para el desarrollo de neoformaciones en niños preescolares. Tesis de Maestría, Benemérita Universidad Autónoma de Puebla, México.

González Moreno, C. X. (2009). La utilización de la actividad de juego temático de roles sociales en la formación del pensamiento reflexivo en preescolares. Tesis de Maestría, Pontificia Universidad Javeriana, Bogotá, Colombia.

González Moreno, C. X., Solovieva, Y. \& Quintanar Rojas, L. (2009). La actividad de juego temático de roles en la formación del pensamiento reflexivo en preescolares. Magis. Revista Internacional de Investigación en Educación, 2 (3), 173-190.

Lázaro, E. (2001). Análisis comparativo de dos evaluaciones: neuropsicológica y psicológica en niños de 6 y 7 años de escuelas privadas y rurales. Tesis de Licenciatura, Benemérita Universidad Autónoma de Puebla, México.

Lázaro, E., Solovieva, Y., Cisneros, N. \& Quintanar, L. (2009). Actividades de juego y cuento para el desarrollo psicológico del niño preescolar. Revista Internacional Magisterio, 37, 80-85.

LeDoux, J. E. (1996). The emotional brain: The mysterious underpinnings of emotional life. New York: Simon $\&$ Schuster.

Leontiev, A. N. (1975). Actividad, conciencia y personalidad. Moscú: Universidad Estatal de Moscú.

Lotman, Y. M. (2003). La Dama de Pique y el tema de juego de cartas en la literatura rusa de los principios del siglo XIX. En Pushkin (pp. 786-814). San Petersburgo: Arte-San Petersburgo.

Obukhova, L. F. (1995). Psicología infantil. Teorías, hechos, problemas. Moscú: Trivola. 
Ocampo, E. (1997). Alternativas pedagógicas posibilitadoras de nuevas formas de aprendizaje y nuevas formas evaluativas. Bogotá: Pontificia Universidad Javeriana.

Pardo, N. (2000). Pensar la escuela para construir sentido. Programa de Fortalecimiento de la Capacidad Científica en la Educación Básica y Media. Bogotá: Universidad Nacional de Colombia.

Quintanar, L. \& Solovieva Y. (2003a). Manual de evaluación neuropsicológica infantil. México: Benemérita Universidad Autónoma de Puebla.

Quintanar, L. \& Solovieva Y. (2003b). Pruebas de evaluación infantil. México: Benemérita Universidad Autónoma de Puebla.

Quintanar, L. \& Solovieva, Y. (2005). Análisis neuropsicológico de los problemas de aprendizaje escolar. Revista Internacional Magisterio, 15, 26-30.

Quintanar, L., Solovieva, Y., Lázaro, E., Mejía, L. \& Eslava, J. (2008). Dificultades en la lectoescritura. Valladolid: Editorial de la Infancia.

Rueda, V. (2001). Características de la esfera voluntaria y verbal en preescolares mayores. Tesis de maestría, Benemérita Universidad Autónoma de Puebla, México.

Salmina, N. G. \& Filimonova, O. G. (2001). Diagnóstico y corrección de la actividad voluntaria en la edad preescolar y escolar. México: Universidad Autónoma de Tlaxcala.
Smith, E. R. (2002). Overlapping mental representations of self and group: Evidence and implications. In J. P. Forgas \& K. D. Williams (Eds.), The social self: Cognitive, interpersonal, and intergroup perspectives (pp. 21-35). Philadelphia: Psychology Press.

Smith, E. R. \& Mackie, D. M. (2007). La Psicología Social (3ª ed.). Philadelphia, PA: Psychology Press.

Solovieva, Y. (2004). El desarrollo intelectual y su evaluación. Una aproximación histórico-cultural. Puebla: Colección Neuropsicológica y Rehabilitación.

Solovieva, Y. \& Quintanar, L. (2005). Análisis neuropsicológico de los problemas en el aprendizaje escolar. Revista Internacional Magisterio, 15, 26-30.

Solovieva, Y. \& Quintanar, L. (2008). Enseñanza de la lectura. Método práctico para la formación lectora. México: Trillas.

Solovieva, Y., Quintanar, L. \& Lázaro, E. (2006). Efectos socioculturales sobre el desarrollo psicológico y neuropsicológico en niños preescolares. Cuadernos Hispanoamericanos de Psicología, 6 (1), 9-20.

Talizina, N. F. (2000). Manual de Psicología Pedagógica. México: Universidad Autónoma de San Luis Potosí.

Vygotsky, L. S. (1995). El problema del desarrollo de las funciones psíquicas superiores. En J. S. Bravo \& L. Kúper de Velasco (Eds. y Trads.), Obras escogidas (Vol. 3, pp. 11-46). Madrid: Visor. 